\title{
Revisión
}

\section{El prematuro tardío: el gran olvidado}

\author{
T. Fernández López ${ }^{a}$, G. Ares Mateos ${ }^{b}$, I. Carabaño Aguado ${ }^{b}$, J. Sopeña Corvinos ${ }^{a}$
}

Publicado en Internet:

5-octubre-2012

Tania Fernández López: taniafer82@yahoo.es
aServicio de Pediatría. Hospital Infanta Elena. Valdemoro, Madrid. España - bservicio de Pediatría. Hospital Rey Juan Carlos. Móstoles, Madrid. España.
Palabras clave:

- Prematuro tardío

- Recién nacido casi a

término $\bullet$ Morbilidad

- Mortalidad
Los recién nacidos prematuros tardíos son aquellos nacidos a una edad gestacional entre la semana $34^{+0}$ y $36^{+6}$. Estos niños tienen tasas de morbilidad más altas que los recién nacidos a término por su relativa inmadurez fisiológica y metabólica, lo que conlleva una estancia hospitalaria prolongada y un aumento del coste económico. Además, la tasa de reingreso y la mortalidad son también mayores que en los nacidos a término.

El objetivo de esta revisión es definir el concepto de "prematuro tardío", describir las complicaciones médicas y riesgos que se encuentran comúnmente en este grupo de niños, presentar las características que les predisponen a una morbimortalidad más alta y sugerir guías para la valoración y manejo de estos pacientes tras el nacimiento.

\section{Late preterm infant: the forgotten patient}

Late preterm infants are born at a gestational age between 34 and 36 weeks and 6 days. These children have higher morbidity than term infants due to their relative physiologic and metabolic immaturity, leading to a longer average birth hospital stay with a higher cost of care. Moreover, the rate of readmission and mortality are also higher than in term infants.

Key words:

The purpose of this review is to define "late-preterm", describe medical complications and risks that

- Late preterm - Near term $\bullet$ Morbidity are commonly found in this group of children, present the characteristics of late-preterm infants that predispose them to higher morbidity and mortality and suggest guidelines for assessment and mana- Mortality gement of these patients after birth.

\section{INTRODUCCIÓN}

Los prematuros tardíos son los nacidos a una edad gestacional (EG) entre las semanas $34^{+0}$ y $36^{+6}$. Estos niños tienen tasas de morbilidad y mortalidad más altas que los recién nacidos a término (EG por encima de 37 semanas) por su relativa inmadurez fisiológica y metabólica, aunque a veces tengan el mismo peso que algunos niños a término ${ }^{1-4}$.

El objetivo de esta revisión es definir el relativamente nuevo concepto de prematuro tardío, des- cribir las complicaciones médicas y los riesgos que se encuentran comúnmente en este grupo de niños y sugerir guías de manejo durante la hospitalización al nacimiento y de después del alta médica.

\section{DEFINICIÓN}

La Organización Mundial de la Salud, la Academia Americana de Pediatría (AAP) y el Colegio Americano de Obstetricia y Ginecología (ACOG) definen nacimiento prematuro como el parto de 
un niño antes de completar las 37 semanas de gestación.

El término "prematuro tardío" fue definido en 2005 por los participantes en el taller titulado "Cómo optimizar la atención y la evolución de los embarazos y los recién nacidos casi término". Se definió como aquel recién nacido entre la semana $34^{+0}$ y la semana $36^{+6}$ de gestación.

Este concepto ha reemplazado al de recién nacido "casi a término", que implicaba que estos niños eran cercanos al término y solo requerían los cuidados neonatales de rutina, subestimando los riesgos que podían tener por su inmadurez.

\section{EPIDEMIOLOGÍA}

En EE. UU., los nacimientos prematuros han aumentado del 10,6\% en 1990 al 12,2\% en 2009, y este aumento se debe sobre todo a los prematuros tardíos ${ }^{5,6}$.

Este cambio se ha atribuido principalmente a las mejoras en el seguimiento obstétrico que detecta las condiciones maternas, placentarias y fetales, dando como resultado un aumento en el número de partos inducidos por indicación médica.

Una de las causas principales de aumento de partos prematuros es el aumento del número de embarazos múltiples ${ }^{6}$. Así, un $60 \%$ de los gemelares nacen prematuros, con una EG media de $35^{+2}$. Además, los embarazos múltiples son cada vez más numerosos debido, por un lado, al incremento de madres mayores 30 años, que dan lugar a más embarazos gemelares de forma espontánea que las mujeres más jóvenes; y, por otro lado, al incremento de las técnicas de reproducción asistida ${ }^{6}$. Se estima que la contribución de las técnicas de reproducción asistida al aumento de la tasa de prematuridad es de aproximadamente un $10 \%$.

Como se ha mencionado previamente, en los países desarrollados se han producido mejoras en el seguimiento obstétrico gracias a los avances en la ecografía prenatal y la monitorización fetal. Este incremento del seguimiento es paralelo a un au- mento de la tasa de nacimientos inducidos por motivos médicos. Así, en EE. UU., estos nacimientos han aumentado de un 29\% en 1992 a un $41 \%$ de todos los recién nacidos en 2002.

Otros factores que han provocado el aumento de los partos pretérmino y, particularmente, de los prematuros tardíos, son condiciones maternas como la mayor edad media de las madres y la epidemia de sobrepeso existente en EE. UU. (que aumenta las complicaciones del embarazo como hipertensión arterial, diabetes gestacional, etc.).

Una cuestión importante, sobre todo pensando en la prevención de los partos prematuros tardíos, es la determinación adecuada de la EG. Algunos prematuros tardíos lo son como resultado de partos inducidos de fetos que, incorrectamente, se pensaba que habían llegado a término. Así, la inducción electiva llevada a cabo por motivos no médicos debe evitarse por el riesgo aumentado de morbilidad. No obstante, si va a realizarse, es de extrema importancia asegurar que la EG es adecuada para prevenir el parto prematuro y solo debería realizarse si la EG es al menos de 39 semanas $^{7}$.

\section{MORBIMORTALIDAD}

Los prematuros tardíos tienen una tasa elevada de morbimortalidad, comparada con los recién nacidos a término. En general, tienen tasas más altas de hospitalización al nacimiento, de reingreso en el periodo neonatal y durante el primer año de vida y corren más riesgo de afectación en el neurodesarrollo a largo plazo.

\section{Hospitalización al nacimiento}

Los prematuros tardíos tienen una tasa de morbilidad de seis a siete veces mayor que los nacidos a término ${ }^{8}$. Este riesgo aumenta significativamente en niños con otros factores de riesgo asociados.

Los prematuros tardíos, comparados con recién nacidos a término, implican un coste muy alto. Es un estudio retrospectivo que analizaba datos de una cohorte de neonatos con seguro privado en EE. UU., los prematuros tardíos tenían una estancia media 
hospitalaria más alta (8,8 frente a 2,2 días) con un coste económico casi diez veces mayor (26 054 \$ frente a $2061 \$)^{9}$.

Son fisiológica y metabólicamente inmaduros y se comportan como tal. Los principales problemas que podemos encontrar son hipotermia, hipoglucemia (6,8 frente a $0,4 \%$ ), dificultad respiratoria (4,2 frente a 0,1\%), apnea, hiperbilirrubinemia (18 frente a $2,5 \%$ ) y dificultades de alimentación ${ }^{10}$.

\section{Hipotermia}

Los prematuros tardíos son más susceptibles a la hipotermia comparados con los nacidos a término, ya que tienen menor capacidad para generar calor a partir del tejido adiposo pardo, menores depósitos de tejido adiposo blanco para el aislamiento del frío y pierden calor rápidamente por su elevada ratio superficie/peso ${ }^{3,10,11}$. Además, presentan una inmadurez de la función hipotalámica termorreguladora.

\section{Hipoglucemia}

El riesgo de hipoglucemia es tres veces mayor que en los nacidos a término, y la incidencia aumenta con la menor $\mathrm{EG}^{11}$. Se produce porque la respuesta metabólica es insuficiente ante el corte brusco de suministro materno de glucosa tras el nacimiento. En los prematuros tardíos, la glucogenólisis hepática y la lipólisis del tejido adiposo son inmaduras y también existe déficit de la gluconeogénesis y cetogénesis hepática.

\section{Morbilidad respiratoria}

El riesgo de presentar morbilidad respiratoria es mayor en los prematuros tardíos que en los nacidos a término. Se ha visto que existe mayor riesgo de dificultad espiratoria (4,2 frente a 0,1\%), que la tasa de admisión en unidades de cuidados intensivos neonatales es mayor (36,5 frente a 7,2\%), así como la admisión con compromiso respiratorio $(10,5 \text { frente a } 1,1 \%)^{12}$. Además, los prematuros tardíos requieren mayor grado de reanimación en la sala de partos que los nacidos a término. A excep- ción de la intubación por meconio, el grado de reanimación requerido decrece con la mayor EG hasta la semana 39.

El riesgo aumentado de morbilidad respiratoria en los prematuros tardíos se debe a la inmadurez de la estructura pulmonar, que continúa desarrollándose hasta la semana 36. Además, en algunos prematuros aparecen deficiencias funcionales del surfactante, lo que aumenta el riego de dificultad respiratoria, especialmente en aquellos hijos de madres que no han recibido corticoides prenata-

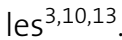

La taquipnea transitoria del recién nacido y la hipertensión pulmonar persistente son otras causas de dificultad respiratoria observadas en recién nacidos prematuros tardíos ${ }^{3,10,14}$.

\section{Apnea}

La incidencia encontrada de apnea en prematuros tardíos (4-7\%) es mayor que en los nacidos a término (1-2\%) y también tienen mayor riesgo de muerte súbita.

\section{Hiperbilirrubinemia}

Debido a la inmadurez y al retraso en el desarrollo de las vías de conjugación de bilirrubina, los prematuros tardíos tienen el doble de riesgo de sufrir concentraciones elevadas de bilirrubina indirecta a los cinco días de vida. Las dificultades de alimentación también pueden conducir a un retraso en la recirculación enterohepática de la bilirrubina. Así, las concentraciones de bilirrubina sérica van a ser más altas, su duración más prolongada y el pico suele ser más tardío, entre el quinto y el séptimo día de vida $2,10,15$.

Además, ante la misma cifra de bilirrubina, el riesgo de daño cerebral inducido por bilirrubina y el de kernicterus es mayor en el prematuro tardío que en el nacido a término, debido a la relativa inmadurez de la barrera hematoencefálica, las concentraciones más bajas de proteína transportadora de bilirrubina y el riesgo aumentado de enfermedad concurrente ${ }^{15,16}$. 


\section{Dificultades de alimentación}

La lactancia materna es la alimentación óptima para estos niños, por los beneficios que tiene para madre e hijo. Sin embargo, a menudo requieren apoyo y monitorización adicional en comparación con los recién nacidos a término, debido a la inmadurez de la coordinación orobucal y de los mecanismos de succión-deglución de estos bebés. Los problemas para establecer una buena alimentación parecen ser el factor que más contribuye al riesgo aumentado de reingreso por deshidratación que sufren estos pacientes.

\section{Reingreso hospitalario}

La tasa de reingreso hospitalario es de dos a tres veces mayor en los prematuros tardíos ${ }^{2,9,17}$. Particularmente, tienen un riesgo de reingreso tres veces mayor en los primeros 15 días tras el nacimiento ${ }^{9}$. Las causas más frecuentes de reingreso son ictericia, dificultades de alimentación, escasa ganancia ponderal, deshidratación y apnea.

Los trastornos respiratorios (incluida la bronquiolitis) y gastrointestinales son las causas más comunes de reingreso tardío en el primer año de vida. Un estudio de California observó que la cohorte más grande de niños que reingresaron al menos una vez en el primer mes de vida fue de recién nacidos de 35 semanas de gestación, debido principalmente a infecciones respiratorias ${ }^{18}$. El coste de estos reingresos fue de 92,9 millones de dólares.

La AAP ha identificado factores de riesgo para el reingreso que pueden ayudarnos en la práctica clínica. Estos son ser el primer hijo, alimentación con lactancia materna exclusiva al alta y ser hijo de madre con complicaciones en el puerperio.

\section{Complicaciones a largo plazo}

Varios estudios sugieren que los prematuros tardíos tienen mayor riesgo de morbilidad en el neurodesarrollo que los nacidos a término ${ }^{18-20}$. Así, existe un riesgo aumentado de parálisis cerebral $(0,43$ frente a $0,14 \%)$ y de retraso mental $(0,81$ frente a $0,49 \%)$. También se ha visto que existe mayor frecuencia de problemas de comportamiento, mayor fracaso escolar y un coeficiente intelectual más bajo ${ }^{21}$. Además, la prematuridad se ha asociado con una mayor frecuencia de problemas de aprendizaje y atención, con mayor frecuencia de trastorno por déficit de atención con hiperactividad. La inmadurez cerebral puede jugar un papel importante en el retraso del neurodesarrollo de estos niños. Se ha visto en autopsias que el peso del cerebro de los prematuros de 34 semanas es el $65 \%$ del peso de un recién nacido a término, tiene menos surcos en su superficie y está menos mielinizado ${ }^{22}$.

\section{Mortalidad}

Aunque el riesgo relativo de mortalidad de los prematuros tardíos frente a los recién nacidos a término es modesto comparado con los prematuros menores de 32 semanas de EG, alrededor del 10\% de todas la muertes neonatales en EE. UU. ocurre en prematuros tardíos.

Los prematuros tardíos tienen mayor riesgo de mortalidad que los término, en EE. UU., esta es hasta tres veces mayor. El riesgo de muerte neonatal aumenta según disminuye la EG y también se ve afectada por otros factores como malformaciones congénitas y crecimiento intrauterino retardado.

\section{MANEJO Y TRATAMIENTO}

\section{Prevención}

Para disminuir la morbimortalidad asociada con la prematuridad tardía, la prevención es uno de los puntos clave. Un paso esencial para disminuir los nacimientos pretérmino es asegurar que no se realizan partos electivos antes de la 39 semanas de EG, a menos que haya una indicación médica ${ }^{23}$.

Además, se necesitaría mejorar el manejo del feto y la madre embarazada en estas gestaciones. Las áreas de investigación en las que se está trabajando para ello incluyen la identificación de tratamientos para disminuir las complicaciones en los prematuros, las mejoras en la precisión a la hora de determinar la EG y la identificación de fetos con riesgo de muerte intrauterina. 


\section{Manejo previo al alta hospitalaria}

Los médicos que se ocupan del cuidado de los recién nacidos prematuros tardíos necesitan conocer sus complicaciones asociadas y saber proveer los cuidados necesarios. Además, antes del alta hospitalaria hay que concienciar a los padres de que su hijo tiene un riesgo aumentado de hiperbilirrubinemia, dificultades de alimentación y deshidratación. La educación debe centrarse en desarrollar la habilidad de los padres para reconocer estos problemas. Es especialmente importante educar a las madres primerizas de los prematuros tardíos y, en ocasiones, esta educación requiere una hospitalización al nacimiento más larga.

La AAP ha establecido las siguientes guías para el alta hospitalaria de los prematuros tardíos ${ }^{8,24}$ :

- Determinar adecuadamente la EG antes del alta y asegurarse de que no hay anomalías o motivos médicos que justifiquen alargar la hospitalización.

- El recién nacido debe demostrar estabilidad fisiológica, demostrando competencia en lo siguiente:

- Termorregulación, definida como temperatura axilar entre 36,5 y $37,4{ }^{\circ} \mathrm{C}$ en una cuna abierta.

- Alimentación, definida como adecuada succión-deglución, respiración adecuada mientras se hace la toma y pérdida de peso que no exceda el $7 \%$ con respecto al peso del nacimiento. Si el niño es amamantado, se debe objetivar antes del alta una adecuada técnica de lactancia.

- Estabilidad cardiorrespiratoria, con constantes vitales estables (frecuencia respiratoria menor de 60 respiraciones por minuto y frecuencia cardiaca entre 100 y 160 latidos por minuto).

- Tránsito intestinal, habiendo realizado al menos una deposición de forma espontánea.

- Deben haberse realizado los cuidados de rutina del recién nacido, tales como pruebas metabólicas, vacuna y profilaxis ocular y antihemorrágica.
- Educación y entrenamiento adecuado de los padres para el cuidado de estos niños y para ser capaces de reconocer problemas como la ictericia, las dificultades de alimentación y la deshidratación.

- Se debe establecer una visita de seguimiento a las 24-48 horas del alta hospitalaria por un pediatra en el ámbito de la Atención Primaria.

\section{CONCLUSIÓN Y PUNTOS CLAVE ${ }^{25-27}$}

- Los recién nacidos prematuros tardíos son aquellos nacidos a una EG entre las semanas 34 y 36 . Tienen tasas de morbilidad más altas que los recién nacidos a término.

- En EE. UU., la tasa de prematuros tardíos es casi del $9 \%$ de todos los nacimientos, habiendo aumentado en la última década debido a los embarazos múltiples y a los partos prematuros médicamente indicados.

- Se estima que los prematuros tardíos tienen siete veces más riesgo de morbilidad durante la hospitalización al nacimiento, en comparación con los recién nacidos a término, lo que conlleva un estancia hospitalaria prolongada y un aumento del coste económico.

- La tasa de reingreso es de dos a tres veces mayor que la de los nacidos a término. Muchos de estos reingresos podrían evitarse mediante una cuidadosa monitorización y apoyo de la lactancia materna durante el ingreso postnatal.

- Parece que los prematuros tardíos tienen un riesgo aumentado de alteraciones en el neurodesarrollo a largo plazo, en comparación con los nacidos a término.

- La tasa de mortalidad de los prematuros tardíos es al menos tres veces mayor que la de los nacidos a término.

- Algunas estrategias para disminuir la morbimortalidad asociada con los prematuros tardíos son la prevención del parto prematuro, el establecimiento de una lactancia materna ade- 
cuada y el tratamiento y prevención de las complicaciones médicas asociadas con la prematuridad tardía.

\section{CONFLICTO DE INTERESES}

Los autores declaran no presentar conflictos de intereses en relación con la preparación y publicación de este artículo.

\section{BIBLIOGRAFİA}

1. Escobar GJ, Gonzales VM, Armstrong MA, folck BF, Xiong B, Newman TB. Rehospitalizacion for neonatal dehydratation: a nested case-control study. Arch Pediatr Adolesc Med. 2002;156:155-61.

2. Escobar GJ, McCornimick MC, Zupanic JA, Coleman Phox K, Armstrong MA, Greene JD, et al. Unstudied infants: outcomes of moderately premature infants in the neonatal intensive care unit. Arch Dis Child Fetal Neonatal Ed. 2006;91:F238-44.

3. Wang ML, Dorer DJ, Fleming MP, Catlin EA. Clinical outcomes of near-term infants. Pediatrics. 2004; $114: 372-6$

4. Kramer MS, Demissie K, Yang H, Platt RW, Sauvé R, ListonR. The contribution of mild and moderate preterm birth to infant mortality. Fetal and Infant Health Study Group of the Canadian Perinatal Surveillance System. JAMA. 2000;284:843-9.

5. Davidoff MJ, Dias T, Damus K, Rusell R, Bettegowsa VR, Dolan S, et al. Changes in the gestational age distribution among U.S. singleton births: impact on rates of late preterm birth, 1992 to 2002. Semin Perinatol. 2006;30:8-15.

6. Martin JA, Hamilton BE, Sutton PD, Ventura SJ, Menacker F. Births: Final data for 2006. Natl Vital Stat Rep. 2009;57:1-101.

7. Engle WA, Kominiarek MA. Late preterm infants, early term infants, and timing of elective deliveries. Clin Perinatol. 2008;35:325-41.

8. Engle WA, Tomashek KM, Wallman C; Committee on Fetus and Newborn, American Academy of Pediatrics. "Late-preterm" infants: a population at risk. Pediatrics. 2007;120:1390-401.

9. Bird TM, Bronstein JM, Hall RW, Lowery CL, Nugent R, Mays GP. Late preterm infants: birth outcomes and

\section{ABREVIATURAS}

AAP: Academia Americana de Pediatría - ACOG: Colegio Americano de Obstetricia y Ginecología • EG: edad gestacional.

health care utilization in the first year. Pediatrics. 2010;126:e311-9.

10. Leone A, Ersfeld P, Adams M, Schiffer PM, Bucher HU, Arlettaz R. Neonatal morbidity in singleton late preterm infants compared with full-term infants. Acta Paediatr. 2012;101:e6-10.

11. Laptook A, Jackson GL. Cold stress and hypoglycemia in the late preterm ("near-term") infant: impact on nursery of admission. Semin Perinatol. 2006;30:24-7.

12. Rubaltelli FF, Bonafe L, Tangucci M, Spagmolo A, Dani C. Epidemiology of neonatal acute respiratory disorders. A multicenter study on incidence and fatality rates of neonatal acute respiratory disorders according to gestational age, maternal age, pregnancy complications and type of delivery. Italian Group of Neonatal Pneumology. Biol Neonate. 1998;74:7-15.

13. Jain L. Respiratory morbidity in late-preterm infants: prevention is better than cure! Am J Perinatol. 2008;25:75-8.

14. Engle WA; American Academy of Pediatrics Committee on Fetus and Newborn. Surfactant-replacement therapy for respiratory distress in the preterm and term neonate. Pediatrics. 2008;121:419-32.

15. Bhutani VK, Maisels MJ, Stark AR, Buonocore G. Management of jaundice and prevention of severe neonatal hyperbilirubinemia in infants $>$ or $=35$ weeks gestation. Neonatology. 2008;94:63-7.

16. Radtke JV. The paradox of breastfeeding-associated morbidity among late preterm infants. J. Obstet gynecol Neonatal Nurs. 2011;40:9-24.

17. Escobar GJ, Joffe S, Gardner MN, Armnstrong MA. Folck BF, Carpenter DM. Rehospitalization in the first two weeks after discharge from the neonatal intensive care unit. Pediatrics. 1999;104:e2.

18. Morse SB, Zheng H, Tang Y, Roth J. Early school-age outcomes of late preterm infants. Pediatrics. 2009; 123:e622-9. 
19. Petrini JR, Dias T, McCormick MC, Massolo MN, Green MS, Escobar GJ. Increased risk of adverse neurological development for late preterm infants. J Pediatr. 2009;154:169-76.

20. Gurka MJ, LoCasale-Crouch J, Blackman JA. Longterm cognition, achievement, socioemotional, and behavioral development of healthy late-preterm infants. Arch Pediatr Adolesc Med. 2010;164:525-32.

21. Moster D, Lie RT, Markestad T. Long-term medical and social consequences of preterm birth. N Engl J Med. 2008;359:262-73.

22. Tomashek KM, Shapiro-Mendoza CK, Davidoff MJ, Petrini JR. Differences in mortality between late-preterm and term singleton infants in the United States, 1995-2002. J Pediatr. 2007;151:450-6.

23. Committee on Obstetric Practice. ACOG committee opinion No. 404 April 2008. Late-preterm infants. Obstet Gynecol. 2008;111:1029-32.
24. Whyte RK. Neonatal management and safe discharge of late and moderate preterm infants. Semin Fetal Neonatal Med. 2012;17:153-8.

25. de Jong $M$, Verhoeven $M$, van Baar AL. School outcome, cognitive functioning, and behaviour problems in moderate and late preterm children and adults: $A$ review. Semin Fetal Neonatal Med. 2012;17:163-9.

26. Shapiro-Mendoza CK, Lackritz EM. Epidemiology of late and moderate preterm birth. Semin Fetal Neonatal Med. 2012;17:120-5.

27. Gouyon JB, lacobelli S, Ferdynus C, Bonsante F. Neonatal problems of late and moderate preterm infants. Semin Fetal Neonatal Med. 2012;17:146-52. 